RAIRO Operations Research

RAIRO Oper. Res. 41 (2007) 455-464

DOI: $10.1051 /$ ro:2007031

\title{
NEWSVENDOR SOLUTIONS WITH GENERAL RANDOM YIELD DISTRIBUTIONS
}

\author{
Scott E. Grasman ${ }^{1}$, ZAKI SARI ${ }^{2}$ AND TeWfiK SARI ${ }^{3}$
}

\begin{abstract}
Most systems are characterized by uncertainties that cause throughput to be highly variable, for example, many modern production processes and services are substantially affected by random yields. When yield is random, not only is the usable quantity uncertain, but the random yield reduces usable capacity and throughput in the system. For these reasons, strategies are needed that incorporate random yield. This paper presents the analysis of the newsvendor model with a general random yield distribution, including the derivation of the optimal order quantity. Results are shown to converge to the basic newsvendor model for the case of perfect yield, and are further demonstrated using the case of general multiplicative random yield. Results have significant impact on both manufacturing and service sectors since the newsvendor model applies to many real-world situations.
\end{abstract}

Keywords. Newsvendor model, operations management, planning and control, random yield.

Mathematics Subject Classification. 90B.

\section{INTRODUCTION AND LITERATURE REVIEW}

Many modern production processes and services are substantially affected by random yields. Semiconductor manufacturers, for instance, often have yields below $50 \%$ and plastics producers often run at around $75 \%$ (see, for example, Gilbert

Received October 9, 2005. Accepted May 25, 2007.

1219 EMSE, University of Missouri - Rolla, Rolla, Missouri 65409-0370, USA; grasmans@umr. edu

${ }^{2}$ Laboratoire d'Automatique de Tlemcen, Faculté des Sciences de l'Ingénieur, Université

Abou Bekr Belkaïd de Tlemcen, Algeria; z_sari@univ-tlemcen.edu

${ }^{3}$ Mathematics, University of Mulhouse, France; t.sari@uha.fr

(c) EDP Sciences, ROADEF, SMAI 2007

Article published by EDP Sciences and available at http://www.rairo-ro.org or http://dx.doi.org/10.1051/ro:2007031 
and Emmons [4], Bohn and Terwiesch [1], and Grasman et al. [6]). Many of these processes are new and production yields are well below the ideal. As more is learned, yield increases, but will never reach 100 percent - or even come close in some cases. Rapidly changing technologies may become obsolete before the process is well understood, or it may not be financially justifiable to correct the yield problem; therefore, in these situations, methods are needed to cope with uncertainty. Yano and Lee [12] points out that while the ultimate goal is to make yields perfect, random yield models are valuable for many reasons. First, results can be used in the short term to help an operation run more efficiently so that efforts can be focused on yield improvement. Second, process improvement and supplier decisions can be assessed more accurately and effectively if system effects of these decisions are modeled accurately and optimized. Third, models can assist in capacity planning decisions when random yield is expected to be a long run concern.

Random yields are not confined to manufacturing industries. Service industries, for example, hotels, airlines, and healthcare facilities, incur large costs due to the random nature of confirmed no-shows not meeting appointments. Consider a hotel, which has a given number of rooms available on a specific date, an airline, which has a given number of seats available for a specific flight, or a healthcare facility, which has a given number of available appointments. Some customers will not show up and also not cancel their reservations/appointments. The question is how many reservations to take - on one hand there is a penalty associated with underbooking, and, on the other hand, there is a penalty associated with overbooking. These types of problems may be considered newsvendor problems with random yield.

The subject of process yields has received considerable attention, which is not surprising considering its significance across manufacturing and service industries. Numerous papers have been written regarding production/inventory models with random yield; however, few deal with the generalized system presented here. Yano and Lee [12] provides a comprehensive review of the literature on quantitativelyoriented approaches for determining lot sizes when production or procurement yields are random. Issues discussed relate to the modeling of costs, yield uncertainty, and performance. Grosfeld-Nir and Gerchak [7] provides a review of recent advances in models, analytic results, and insights pertaining to random yield production environments. Khouja [10] builds a taxonomy of newsvendor-related literature including extensions to random yields.

Although many classical models, including newsvendor models, provide optimal results for a wide variety of problems, they cannot be directly applied to systems with random yield. Existing classical production approaches may be modified to consider average yield rates by simply adjusting the production rates and normalizing the production costs per unit to account for average yield losses, but this approach is insufficient. Such approaches do not capture the fact that, in the presence of random yield, if the inventory level falls below a critical level, the amount produced depends on yield variability (see, Henig and Gerchak [8]); thus these approximations yield optimal results only if the yield is deterministic. If the 
quantity started is determined simply by allowing for average yield rate, demand will only be met a fraction of the time and large shortage costs will generally be incurred. Bollapragada and Morton [2] discuss these approaches at length.

In the authors opinion, the most closely related paper is Gerchak, Vickson and Parlar [3], which presents a dynamic version of the problem and proves certain structural results. The main difference is that yield is represented using a multiplicative factor, $Y_{Q}=Q U$, where $U$ is a nonnegative random variable. This assumption is a special case of the results presented in this paper and is highlighed in Section Four. Although Gerchak, Vickson and Parlar extend their model to allow for the initial inventory to be positive in a multiperiod model, they again do not consider general random yield. It should be noted that allowing for initial inventory does not allow the conditions for myopic solutions to be satisfied; therefore, a challenging extension to this paper would be to address this issue in the context of general random yield.

Thus, this paper presents the analysis of the newsvendor model with a general random yield distribution, and is organized as follows. Section Two provides an overview of random yield distributions, Section 3 provides the formulation of the newsvendor model with general random yield and demonstrates the convergence to the basic newvendor solution for the case of perfect yield. Section Four further demonstrates the model for general multiplicative yield. Finally, Section Five provides conclusions and extensions.

\section{OVERVIEW OF RANDOM YIELD DISTRIBUTIONS}

The most important aspect of random yield production models is the distribution of the yield. Yield distributions may or may not be independent of the production quantity. It is important to note that the yield rate may be independent of the production quantity, however, the number of defects produced (or the actual yield) is almost certainly dependent on the production quantity. Various random yield distributions are now detailed.

Deterministic: A known fraction of the production quantity is defective. Heyman and Sobel [9](p. 79) have shown that problems with deterministic yield may be modeled as perfect yield systems.

Multiplicative: A random multiple, $m$, of the production quantity, $Q$, is defective. Thus,

$Y=Q m$ where, $m$ follows a given random distribution.

Also called stochastically proportional common-cause yield, the distribution of the fraction of good items produced is specified (and multiplied by $Q$ to get the number of good units). This approach allows for specification of both the mean and variance of the fraction of good items, but forces the yield rate to be independent of the number produced. This modeling approach applies when large batches are produced. Thus, for a uniform multiplier where $m \sim$ Uniform $[0,1], Y=Q m, E[Y]=\frac{1}{2} Q$ and 
$E\left[Y^{2}\right]=\frac{1}{3} Q^{2}$. Note that the most common multiplier is distributed uniformly, but should not be confused with uniformly distributed yield, where the number of good items is equally distributed.

Uniform: The number of good items is equally distributed between 0 and $Q$. Thus,

$$
P\left(Y_{Q}=j\right)=\frac{1}{Q+1} \text { for } j=0,1,2, \ldots, \mathrm{Q} .
$$

Binomial: Each item produced has a Bernoulli distributed probability of being defective, i.e., occurrences of defects during a production run are independent and identically distributed. The creation of good units is a Bernoulli process, hence the number of good units in a batch of size $Q$ is binomially distributed with parameters $Q$ and $p$, where $p$ is the probability of producing a good unit. One advantage of this model is that only the value of $p$ needs to be specified. One disadvantage is that is does not allow for specification of variance. The expected value of the fraction of good units produced remains constant $(p)$, but the variance of the fraction of good units, $\frac{p(1-p)}{Q}$, decreases with $Q$. This modeling approach is appropriate for systems that are in control for long periods of time since each unit produced may be considered independent of the previous unit. Thus,

$$
P\left(Y_{Q}=j\right)=\left(\begin{array}{c}
Q \\
j
\end{array}\right) p^{j}(1-p)^{Q-j} \text { for } j=1,2, \ldots, \mathrm{Q} .
$$

A significant contrast between binomial and multiplicative yield is independence of the individual yields exhibited by the binomial model, versus perfect correlation as implied by the multiplicative model. Accordingly, in the binomial model, the variance of the yield rate (fraction nondefective) diminishes as batch size increases, while in the proportional model it is not affected by the batch size.

Lot size dependent: The most common form of lot size dependent yield is when the yield decreases due to deteriorating production processes. If no defects are produced when the process is in control, the shift to out of control is immediately signaled by the presence of a defect. The distribution of the fraction of good items produced is dependent on the number of items produced, and requires the distribution of the time until the process goes out of control (usually exponential) or the probability that the process will go out of control after each unit has been produced (leading to a geometric distribution). Let $\beta$ denote the probability that the process remains in control after the completion of each unit. If the time until the process goes out of control is exponential, then $\beta=\mathrm{e}^{-\frac{\lambda}{\mu}}$ where $\mu$ is the production rate and $\frac{1}{\lambda}$ is the mean time that the process remains in control. Then the number of units produced until the next failure is distributed according to the geometric distribution. The distribution of the fraction of good items may also increase with the number of units produced. This may occur 
due to setup, start up and learning, and may be modeled in the opposite manner to a deteriorating process, by assuming that the process is out of control until the first (or first $n$ ) good units are produced.

\section{MODEL FORMULATION}

This section presents the model formulation for the newsvendor model with generalized random yield. Notation used throughout the paper is presented first, followed by derivation of the expected total cost and optimal order quantity. The section concludes by demonstrating that the results simplify to the standard newsvendor model for the case of perfect yield.

\subsection{Notation}

The following notation will be used throughout the paper.

$$
\begin{aligned}
X & =\text { Random Demand } \\
F(x) & =\text { CDF of } X \\
f(x)=\frac{d}{\mathrm{~d} x} F(x) & =\text { PDF of } X \\
Q & =\text { Order quantity } \\
Y & =\text { Random Yield variable } \\
g_{Q}(y) & =\text { PDF of } Y \\
P & =\text { Random Percentage of Defects } \\
\pi_{Q}(p) & =\text { PDF of } P \\
C_{o} & =\text { Penalty Cost for Overage } \\
C_{s} & =\text { Penalty Cost for Shortage } \\
C(Q) & =\text { Expected Total Cost }
\end{aligned}
$$

\subsection{TOTAL COST AND OPTIMAL ORDER QUANTITY}

In order to define the function for expected total cost, define sets $O=\{(x, y)$ : $x \leq y\}$ and $U=\{(x, y): x \geq y\}$. Figure 1 graphically displays the outcome space for realized yield and demand.

The expected overage and underage can be calculated by integrating over the sets $O$ and $U$, respectively, thus

$$
\begin{aligned}
C(Q) & =\int_{O} C_{o}(y-x) f(x) \mathrm{d} x g_{Q}(y) \mathrm{d} y+\int_{U} C_{s}(x-y) f(x) \mathrm{d} x g_{Q}(y) \mathrm{d} y \\
& =\int_{y=0}^{Q}\left[C_{o} \int_{x=0}^{y}(y-x) f(x) \mathrm{d} x g_{Q}(y)+C_{s} \int_{x=y}^{\infty}(x-y) f(x) \mathrm{d} x g_{Q}(y)\right] \mathrm{d} y \\
& =\int_{y=0}^{Q} g_{Q}(y)\left[C_{o} \int_{x=0}^{y}(y-x) f(x) \mathrm{d} x+C_{s} \int_{x=y}^{\infty}(x-y) f(x) \mathrm{d} x\right] \mathrm{d} y . \quad(1)
\end{aligned}
$$




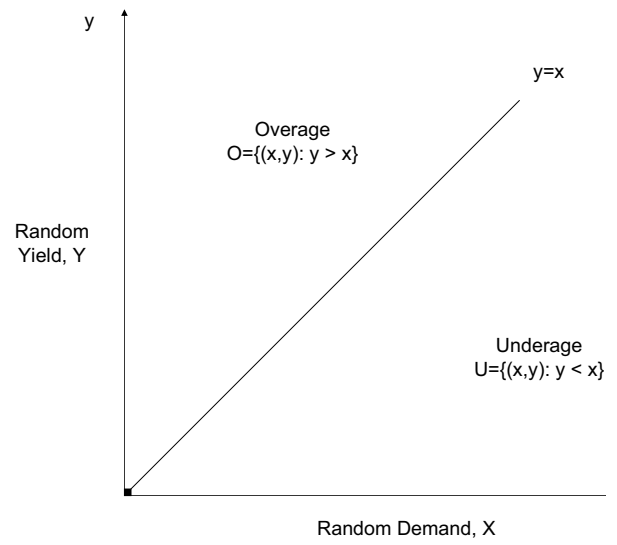

Figure 1. Realization of yield and demand.

Taking the derivative of Equation (1) with respect to $Q$, we have

$$
\begin{gathered}
\frac{\mathrm{d}}{\mathrm{d} Q} C(Q)=\int_{y=0}^{Q} \frac{\mathrm{d}}{\mathrm{d} Q} g_{Q}(y) L(y) \mathrm{d} y+g_{Q}(Q) L(Q), \text { where } \\
L(y)=C_{o} \int_{x=0}^{y}(y-x) f(x) \mathrm{d} x+C_{s} \int_{x=y}^{\infty}(x-y) f(x) \mathrm{d} x .
\end{gathered}
$$

Finally, since $\int_{y=0}^{Q} g_{Q}(y) \mathrm{d} y=1 \Rightarrow \int_{y=0}^{Q} \frac{\mathrm{d}}{\mathrm{d} Q} g_{Q}(y) \mathrm{d} y+g_{Q}(Q)=0$,

$$
\frac{\mathrm{d}}{\mathrm{d} Q} C(Q)=\int_{y=0}^{Q} \frac{\mathrm{d}}{\mathrm{d} Q} g_{Q}(y)[L(y)-L(Q)] \mathrm{d} y
$$

$C(Q)$ is a convex function, and an expression for the optimal order quantity may be obtained by setting Equation (3) equal to zero and solving for $Q^{*}$; thus the equation for $Q^{*}$ is

$$
\begin{gathered}
\int_{y=0}^{Q^{*}} \frac{\mathrm{d}}{\mathrm{d} Q} g_{Q^{*}}(y)\left[C_{o} \int_{x=0}^{y}(y-x) f(x) \mathrm{d} x+C_{s} \int_{x=y}^{\infty}(x-y) f(x) \mathrm{d} x\right. \\
\left.-\quad C_{o} \int_{x=0}^{Q^{*}}\left(Q^{*}-x\right) f(x) \mathrm{d} x-C_{s} \int_{x=Q^{*}}^{\infty}\left(x-Q^{*}\right) f(x) \mathrm{d} x\right] \mathrm{d} y=0 .
\end{gathered}
$$


Rearranging, the equation for $Q^{*}$ becomes

$$
\begin{aligned}
& \left(C_{o}+C_{s}\right) \int_{y=0}^{Q^{*}} \frac{\mathrm{d}}{\mathrm{d} Q} g_{Q^{*}}(y) \int_{x=y}^{Q^{*}}(x-y) f(x) \mathrm{d} x \mathrm{~d} y \\
= & \int_{y=0}^{Q^{*}}\left(Q^{*}-y\right) \frac{\mathrm{d}}{\mathrm{d} Q} g_{Q^{*}}(y) \mathrm{d} y\left(\left(C_{o}+C_{s}\right) \int_{0}^{Q^{*}} f(x) \mathrm{d} x-C_{s}\right) .
\end{aligned}
$$

\subsection{CASE OF PERFECT YIELD}

For the case of perfect yield, the PDF $g_{Q}(y)$ for $Y$ is the delta function $\delta_{Q}$. Thus

and

$$
\int_{y=0}^{Q^{*}} \frac{\mathrm{d}}{\mathrm{d} Q} g_{Q^{*}}(y) \int_{x=y}^{Q^{*}}(x-y) f(x) \mathrm{d} x \mathrm{~d} y=0,
$$

$$
\int_{y=0}^{Q^{*}}\left(Q^{*}-y\right) \frac{\mathrm{d}}{\mathrm{d} Q} g_{Q^{*}}(y) \mathrm{d} y=1 .
$$

Therefore, Equation (4) reduces to

$$
\left(C_{o}+C_{s}\right) \int_{0}^{Q^{*}} f(x) \mathrm{d} x-C_{s}=0
$$

which corresponds to the well-known optimal order for the basic newsvendor model.

\section{NEWSVENDOR SOLUTIONS FOR MULTIPLICATIVE YIELD}

The previous section presents results for general random yield distributions. To demonstrate the applicability, the analysis is continued in this section for the case of multiplicative random yield, which is commonly found in literature and practice (including Gerchak et al. [3]). While this sections assumes multiplicative random yield, no assumption is made regarding the independence of the multiplicative factor, $p$, from the quantity, $Q$.

Let $y=(1-p) Q$, then

$$
\begin{aligned}
C(Q)= & \int_{p=0}^{1} Q g_{Q}((1-p) Q)\left[C_{o} \int_{x=0}^{(1-p) Q}[(1-p) Q-x] f(x) \mathrm{d} x\right. \\
& \left.+C_{s} \int_{x=(1-p) Q}^{\infty}[x-(1-p) Q] f(x) \mathrm{d} x\right] \mathrm{d} p .
\end{aligned}
$$

Notice that $Q g_{Q}((1-p) Q)=\pi_{Q}(p)$. Indeed, since $Y=(1-P) Q$, we have

$$
\mathbb{P}(Y \leq y)=\mathbb{P}(P \geq 1-y / Q)=\int_{1-y / Q}^{1} \pi_{Q}(\alpha) \mathrm{d} \alpha=\int_{1}^{y} \frac{1}{Q} \pi_{Q}(1-\beta / Q) \mathrm{d} \beta,
$$


thus,

$$
g_{Q}(y)=\frac{1}{Q} \pi_{Q}\left(1-\frac{y}{Q}\right) \Rightarrow Q g_{Q}((1-p) Q)=\pi_{Q}(p) .
$$

Finally, the expected total cost may be given by

$$
\begin{aligned}
C(Q)= & \int_{p=0}^{1} \pi_{Q}(p)\left[C_{o} \int_{x=0}^{(1-p) Q}[(1-p) Q-x] f(x) \mathrm{d} x\right. \\
& \left.+C_{s} \int_{x=(1-p) Q}^{\infty}[x-(1-p) Q] f(x) \mathrm{d} x\right] \mathrm{d} p .
\end{aligned}
$$

\subsection{OPTIMAL ORDER QUANTITY EXPRESSION}

Since $C(Q)$ is a convex function, we may find an expression for the optimal order quantity by setting $\frac{\mathrm{d}}{\mathrm{d} Q} C(Q)=0$. From (6) we obtain

$$
\begin{gathered}
\frac{\mathrm{d}}{\mathrm{d} Q} C(Q)=R(Q)+S(Q), \text { where } \\
R(Q)=\int_{p=0}^{1} \frac{\mathrm{d}}{\mathrm{d} Q} \pi_{Q}(p)\left[C_{o} \int_{x=0}^{(1-p) Q}[(1-p) Q-x] f(x) \mathrm{d} x\right. \\
\left.+C_{s} \int_{x=(1-p) Q}^{\infty}[x-(1-p) Q] f(x) \mathrm{d} x\right] \mathrm{d} p, \text { and } \\
S(Q)=\int_{p=0}^{1} \pi_{Q}(p)\left[C_{o} \int_{x=0}^{(1-p) Q}(1-p) f(x) \mathrm{d} x-C_{s} \int_{x=(1-p) Q}^{\infty}(1-p) f(x) \mathrm{d} x\right] \mathrm{d} p
\end{gathered}
$$

Notice that $S(Q)$ can be rewritten as

$$
S(Q)=\left(C_{o}+C_{s}\right) \int_{p=0}^{1} \pi_{Q}(p) \int_{x=0}^{(1-p) Q}(1-p) f(x) \mathrm{d} x \mathrm{~d} p-C_{s}\left(1-\int_{0}^{1} p \pi_{Q}(p) \mathrm{d} p\right) .
$$

Setting $\frac{\mathrm{d}}{\mathrm{d} Q} C\left(Q^{*}\right)=0$ leads to the following equation from which the optimal order quantity $Q^{*}$ can be determined.

$$
\begin{aligned}
R\left(Q^{*}\right)+\left(C_{o}+C_{s}\right) \int_{p=0}^{1} \pi_{Q^{*}}(p) \int_{x=0}^{(1-p) Q^{*}}(1-p) f(x) \mathrm{d} x \mathrm{~d} p & \\
& -C_{s}\left(1-\int_{0}^{1} p \pi_{Q^{*}}(p) \mathrm{d} p\right)=0
\end{aligned}
$$

\subsubsection{Special cases}

Special cases of (1) independent yield and (2) perfect yield are now presented. Since perfect yield is a special case of independent yield, it is presented last. 
Case 1. $\pi_{Q}(p)=\pi(p)$

If the PDF $\pi_{Q}(p)=\pi(p)$ of $P$ is independent of the order quantity $Q$ we have $R(Q)=0$ and Equation (8) reduces to

$$
\int_{p=0}^{1} \pi(p) \int_{x=0}^{(1-p) Q^{*}}(1-p) f(x) \mathrm{d} x \mathrm{~d} p=\left(1-\int_{0}^{1} p \pi(p) \mathrm{d} p\right) \frac{C_{s}}{C_{o}+C_{s}} .
$$

Integrating first with respect to $p$, then with respect to $x$ we have

$$
\int_{x=0}^{Q^{*}} f(x) \int_{p=0}^{1-x / Q^{*}}(1-p) \pi(p) \mathrm{d} p \mathrm{~d} x=\left(1-\int_{0}^{1} p \pi(p) \mathrm{d} p\right) \frac{C_{s}}{C_{o}+C_{s}} .
$$

This result corresponds to previous results (Shih [11], Eq. (19)).

Case 2. $Y=Q$

The case of perfect yield can be obtained as a particular case of Equation (9). In this case, $\pi(p)$ is a Dirac Function, and

$$
\begin{aligned}
\int_{p=0}^{1-x / Q^{*}}(1-p) \pi(p) \mathrm{d} p & =1, \text { and } \\
\int_{0}^{1} p \pi(p) \mathrm{d} p & =0 .
\end{aligned}
$$

Thus, Equation (9) reduces to

$$
\begin{aligned}
\int_{x=0}^{Q^{*}} f(x) \mathrm{d} x & =\frac{C_{s}}{C_{o}+C_{s}} \\
\Leftrightarrow \quad Q^{*} & =F^{-1}\left(\frac{C_{s}}{C_{o}+C_{s}}\right) .
\end{aligned}
$$

Again, it can be seen that Equation (10) corresponds to the well-known optimal order quantity for the basic newsvendor model.

\section{Conclusions}

This paper presents the analysis of the newsvendor model with a general random yield distribution, including the derivation of the optimal order quantity. Results are shown to converge to the basic newsvendor model for the case of perfect yield, and are further demonstrated using the case of general multiplicative random yield. Results have significant impact on both manufacturing and service sectors since the newsvendor model applies to many real-world situations, thus improving the decision making capabilities of complex system environments. Additionally, the integration and implementation of this work in the form of common elements which may be applied to a variety of applications with consistent managerial implications will lead to cost reductions, increased profits, and improved 
quality. The results will have application in the areas of manufacturing, as well as service industries, such as hotels, airlines, and healthcare. Extensions of the model include constrained multiple products (see, Grasman [5]), multi-echelon systems, and others. As previously mentioned, a challenging extension relates to the inclusion of initial inventory.

Acknowledgements. The lead author acknowledges the Missouri Research Board for support of research on random yield systems. Additionally, the authors acknowledge the educational partnership supported by the Unites States Department of State (Grant S-ECAAS-02-GR-281 PS) that made this collaborative effort possible.

\section{REFERENCES}

[1] R.E. Bohn and C. Terwiesch, The Economics of Yield Driven Processes. J. Oper. Manage. 19 (1999) 41-59.

[2] S. Bollapragada, and T.E. Morton, Myopic Heuristics For the Random Yield Problem. Oper. Res. 47 (1999) 713-722.

[3] Y. Gerchak, R.G. Vickson and M. Parlar, Periodic Review Production Models with Variable Yield and Uncertain Demand. IIE Trans. 20 (1988) 144-150.

[4] S. Gilbert and H. Emmons, Managing a Deteriorating Process in a Batch-Production Environment. IIE Trans. 27 (1993) 233-243.

[5] S.E. Grasman, Capacitated Multiple Product Systems with Random Yield. Working Paper.

[6] S.E. Grasman, T.L. Olsen and J.R. Birge, Setting Basestock Levels in Multiproduct Systems with Setups and Random Yield. IIE Trans. (to appear).

[7] A. Grosfeld-Nir and Y. Gerchak, Multiple Lotsizing in Production to Order with Random Yields: A Review of Recent Advances. Ann. Oper. Res. 26 (2004) 43-69.

[8] M. Henig and Y. Gerchak, The Structure of Periodic Review Policies in the Presence of Random Yield. Oper. Res. 38 (1990) 634-643.

[9] D.P. Heyman and M.J. Sobel, Stochastic Models in Operations Research, Vol. II: Stochastic Optimization. McGraw-Hill (1984).

[10] M. Khouja, The Single-Period (Newsvendor) Problem: Literature Review and Suggestions for Future Research. OMEGA Int. J. Manag. Sci. 27 (1999) 573-553.

[11] W. Shih, Optimal Inventory Policies When Stockouts Result From Defective Products. Int. J. Prod. Res. 18 (1980) 677-686.

[12] C.A. Yano and H.L. Lee, Lot Sizing with Random Yields: A Review. Oper. Res. 43 (1995) 311-334. 